This item was submitted to Loughborough's Research Repository by the author.

Items in Figshare are protected by copyright, with all rights reserved, unless otherwise indicated.

\title{
Experimental investigation of the acoustic black hole effect for flexural waves in tapered plates
}

\section{PLEASE CITE THE PUBLISHED VERSION}

http://dx.doi.org/10.1016/j.jsv.2006.07.035

PUBLISHER

(C) Elsevier

VERSION

AM (Accepted Manuscript)

LICENCE

CC BY-NC-ND 4.0

\section{REPOSITORY RECORD}

Krylov, Victor V., and Edward Winward. 2013. "Experimental Investigation of the Acoustic Black Hole Effect for Flexural Waves in Tapered Plates". figshare. https://hdl.handle.net/2134/11847. 
This item was submitted to Loughborough's Institutional Repository (https://dspace.lboro.ac.uk/) by the author and is made available under the following Creative Commons Licence conditions.

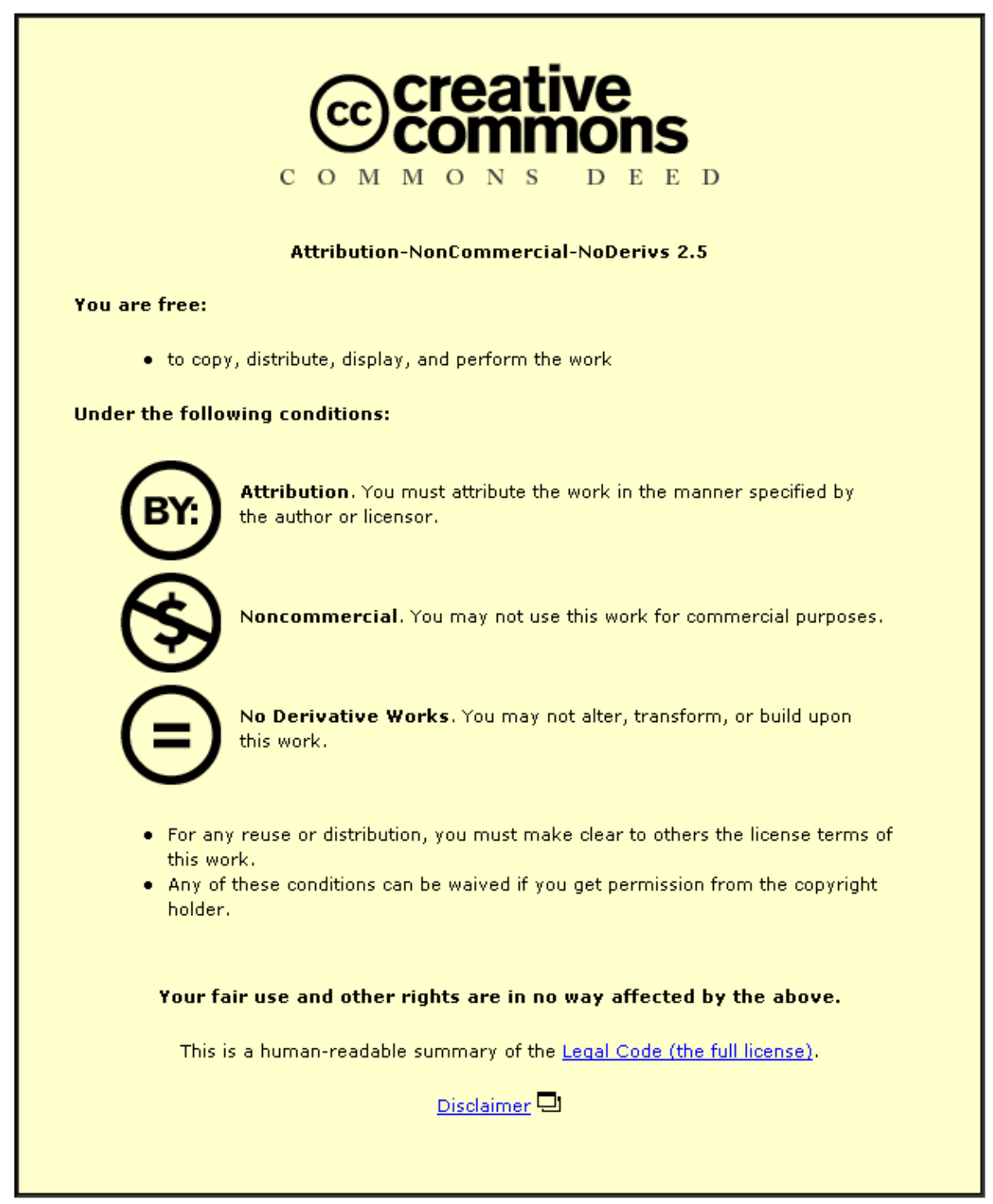

For the full text of this licence, please go to: http://creativecommons.org/licenses/by-nc-nd/2.5/ 


\title{
Experimental investigation of the acoustic black hole effect
}

\section{for flexural waves in tapered plates}

\author{
V.V. Krylov*, R.E.T.B. Winward, \\ Department of Aeronautical and Automotive Engineering, \\ Loughborough University, \\ Loughborough, Leicestershire LE11 3TU, UK
}

\section{Abstract}

An efficient method of reducing edge reflections of flexural waves in plates or bars based on the 'acoustic black hole effect' has been recently proposed and described theoretically by one of the present authors (V.V.K). The method utilises a gradual change in thickness of a plate or bar, partly covered by thin damping layers, from the value corresponding to the thickness of the basic plate or bar (which is to be damped) to almost zero. The present paper describes the results of the experimental investigation of the damping system consisting of a steel plate of quadratic shape (wedge) covered on one side by a strip of absorbing layer. The results of the measurements of point mobility in such a system show that for a wedge covered by an absorbing layer there is a significant reduction of resonant peaks, in comparison with the uncovered wedge or with the covered plate of constant thickness. Thus, the measurements 
confirm the existence of the acoustic black hole effect for flexural waves and demonstrate the possibility of its use in practice.

Keywords: Acoustic black hole effect, flexural waves, quadratic wedges, damping of resonant vibrations.

\section{Introduction}

The present paper reports the results of the experimental investigation of a damping system comprising a steel wedge of quadratic shape covered on one side by a strip of absorbing layer located at the sharp edge (see Fig. 1). Such a system materialises the efficient method of reducing edge reflections of flexural waves in plates or bars based on the 'acoustic black hole effect' that has been recently proposed and described theoretically in the paper [1] (see also $[2-4])$

It is well-known that damping of resonant flexural vibrations of some engineering structures or their components, such as finite plates or bars, can be achieved by reducing reflections of flexural waves from free edges of the structures. This can be made, for example, by introducing graded impedance interfaces, such as combinations of finite plates of the same thickness but made of different materials, along with the placing of damping materials at the edges [5]. The main difficulty in implementing this approach is to create suitable impedance interfaces.

In contrast to [5], the new method proposed in [1-4] uses a gradual change in plate or bar thickness from the value corresponding to the thickness of the basic plate or bar (which is to 
be damped) to almost zero (Fig.1). In other words, it employs elastic wedges of different shapes as gradual impedance interfaces. The above-mentioned gradual change in thickness of a plate or a bar has to be made according to the special laws that ideally provide zero reflections even for negligibly small material attenuation - the so-called 'acoustic black hole effect'. To make up for real manufactured wedges and to improve the damping one should cover wedge surfaces near edges by thin absorbing layers (films), e.g. by polymeric films. According to the theoretical calculations [1-4], wedges of power-law profile covered by thin absorbing layers can be very efficient damping systems, with flexural wave reflection coefficients as low as 1-3 \%.

The aim of this paper is to present the results of the first experimental investigation of a damping system materialising the acoustic black hole effect. The system consists of a steel wedge of quadratic shape covered on one side by a strip of absorbing layer located at the sharp edge. As will be demonstrated, the results of these experiments show that, in agreement with the theory, a significant reduction of resonant peaks can be observed in a wedge covered by an absorbing layer, in comparison with the uncovered wedge or with the free and covered plates of constant thickness. This implies that vibration damping systems utilising the acoustic black hole effect are efficient and suitable for practical applications.

\section{Background information}

The physics of the 'acoustic black hole effect' for flexural waves can be understood using the geometrical acoustics theory of flexural wave propagation near edges of elastic plates of variable thickness gradually decreasing to zero, i.e. near edges of thin elastic wedges of arbitrary shape [6-11]. The phenomenon in question can take place in the special case of 
wedges having cross sections described by a power law relationship between the local thickness $h$ and the distance from the edge $x: h(x)=\varepsilon x^{m}$, where $m$ is a positive rational number and $\varepsilon$ is a constant [9-11]. In particular, it has been shown that for $m \geq 2$ - in free wedges [9-11], and for $m \geq 5 / 3$ - in immersed wedges [9,10], the flexural waves incident at an arbitrary angle upon a sharp edge of a wedge can become trapped near the very edge and therefore never reflect back. Such wedges of specific power-law shapes thus materialise acoustic 'black holes' for flexural waves. In the case of localised flexural waves propagating along edges of such wedges (these waves are also known as wedge acoustic waves) the phenomenon of acoustic 'black holes' associated with the above-mentioned profiles implies that wedge acoustic wave velocities in such structures become equal to zero $[9,10]$.

The unusual effect of power-low profiles on flexural wave propagation in wedges has been first described in the paper [11]. It was pointed out in that paper that a flexural wave does not reflect from the edge of a quadratically shaped wedge in vacuum $(m=2)$, so that even a negligibly small material attenuation can cause all the wave energy to be absorbed near the edge. Unfortunately, because of the deviations of real manufactured wedges from the ideal power-law shapes, largely due to ever-present truncations of the wedge edges, the reflection coefficients in such free wedges are as high as 50-70 \% [11], so that they can not be used as practical vibration dampers.

To improve the situation, a new approach to utilising the acoustic black hole effect has been proposed, namely to cover sensitive parts of the wedge surfaces by thin damping layers (films) of thickness $\delta$, e.g. by polymeric films [1-4]. Note in this connection that the idea of applying absorbing layers for damping flexural vibrations of plates of constant thickness has been used successfully since the 1950's (see, e.g. [12]). The new aspect of this idea, which is used in the proposed approach, is to apply such absorbing layers to plates of variable 
thickness (or wedges) characterised by the specific power-law geometry to achieve maximum damping.

Two types of wedge geometry can be considered: a symmetric wedge and a nonsymmetric wedge, the latter being bounded by a plain surface at one of the sides (Fig. 1). For each of these cases, either two or only one of the sides can be covered by absorbing layers. Note that non-symmetric wedges are easier to manufacture. They also have the technological advantage in the process of depositing absorbing layers: the latter can be deposited on a flat surface, which is much easier. From the point of view of theoretical description, however, there is no difference between symmetrical and non-symmetrical wedges as long as the geometrical acoustics approximation is concerned and the wedge local thickness $h(x)=\varepsilon x^{m}$ is much less than the flexural wavelength.

To analyse the effect of thin absorbing films on flexural wave propagation in a wedge in the framework of geometrical acoustics approximation one should consider first the effect of such films on flexural wave propagation in plates of constant thickness. The simplest way to approach this problem is to use the already known solutions for plates covered by damping layers characterised by the corresponding energy loss factors $v$ (see e.g. [12]). Using this approach (see [3] for more detail), one can derive the corresponding analytical expressions for the reflection coefficients of flexural waves from the edges of truncated wedges of different power-law and sinusoidal profiles covered by thin absorbing layers. In the paper [4], the analysis has been extended to the case of absorbing layers of arbitrary thickness. This has permitted also to carry out more precise calculations of the reflection coefficients for very low values of wedge truncation lengths $x_{0}$, for which the thin film assumption is no longer valid even for very thin films due to the significant reduction of the wedge local thickness.

Typical results of theoretical calculations of the reflection coefficient $R_{0}$ show that the values of the reflection coefficient are remarkably low (in the range of 1-3\%) for small 
values of $x_{0}[3,4]$. This means that the combination of wedges of power-law profiles with thin damping layers can result in very efficient damping systems for flexural vibrations. The degree of energy loss due to the absorption by the wedge with the damping layer, $A^{2}$, can be calculated from the wave energy conservation law: $A^{2}=1-R_{0}^{2}$. Thus, for the abovementioned low reflection coefficients (1-3 \%) the values of $A^{2}$ are in the range of 99.9199.99\%, which means that most of the energy is being absorbed. Note that almost all absorption of the incident wave energy takes place in the vicinity of the sharp edge of a wedge.

\section{Experimental results}

In this section we present some of the results of the experimental investigations of the damping system consisting of a steel wedge of quadratic shape covered on one side by adhesive strips of absorbing layers of various thickness $\delta$ that were located at the sharp edge of the wedge (see Fig. 2). The wedge dimensions were: $280 \mathrm{~mm}$ (length) and $200 \mathrm{~mm}$ (width). Its thickness at the thick end was $4.5 \mathrm{~mm}$, and the value of the quadratic wedge parameter $\varepsilon$ was $5 \times 10^{-5} \mathrm{~mm}^{-1}$. The mass density of the wedge material was $7900 \mathrm{~kg} / \mathrm{m}^{3}$, with the velocities of longitudinal and shear elastic waves being equal to $5900 \mathrm{~m} / \mathrm{s}$ and $3200 \mathrm{~m} / \mathrm{s}$ respectively. The mass density of the absorbing layer was $1160 \mathrm{~kg} / \mathrm{m}^{3}$, with the velocity of longitudinal waves equal to $1900 \mathrm{~m} / \mathrm{s}$. Measurements of point mobility (also known as driving point admittance) have been carried out in a wide frequency range (100-6500 Hz) for a free wedge, for a wedge covered by adhesive strips of absorbing layers, and for a free and covered plate of constant thickness $h=4.5 \mathrm{~mm}$ having the same length and width as the above-mentioned quadratic wedge. 
Experiments were carried out within the Noise and Vibration Laboratory of the Aeronautical and Automotive Engineering Department at Loughborough University. All signal processing was performed using a HP 3566 FFT analyser. Other equipment included a Ling Dynamic Systems 200 series electromagnetic shaker; a Bruel \& Kjaer Type 8200 force transducer; a Bruel \& Kjaer Type 4374 accelerometer; and ENDEVCO Model 27218 charge amplifier.

A shaker providing the excitation input to the wedge (see Figure 2) was attached to the bottom surface of the wedge using a steel 'stinger'. A Bruel \& Kjaer Type 8200 force transducer was then attached to the end of the 'stinger' using a studded fastener. The force transducer was attached to the plate surface via adhesive to minimise mass loading and improve results' validity.

Support of the wedge and plate used in the study was provided by foam resting on a massive steel frame, as illustrated in Fig. 2. In the case of a rectangular plate, the entire surface of the plate was supported by foam to ensure a uniform reaction. The support of the quadratic wedge necessitated the partial removal of foam support in the proximity of the thin edge to enable the free vibration of this section of the wedge. A small aperture was made in the foam to allow access of the force transducer and 'stinger' assembly.

A random (white noise) signal was generated using the HP analyser. Frequency response analysis was then performed on the force transducer and accelerometer measurements.

The results of measurements of point mobility of a free plate are shown in Fig. 3. These measurements have been made for reference purposes. In particular, they included the examining of the possible effect of the attachment of adhesive absorbing strips at the plate edges on its frequency response. The experiments have shown that the attachment of adhesive strips of absorbing layers of various thickness $\delta$ at any of the plate edges did not cause 
noticeable changes in the plate frequency response functions. For that reason, the results of the measurements in the case of presence of adhesive strips are not shown in Fig. 3.

The results of the measurements of the point mobility of a free quadratic wedge and of the same wedge covered by a strip of thin absorbing layer of thickness $\delta=0.2 \mathrm{~mm}$ are shown in Fig. 4. As one can see, in the wedge covered by an absorbing layer there is a significant reduction of resonant peaks, sometimes by up to $20 \mathrm{~dB}$, in comparison with the uncovered wedge. This can be attributed to the significant reduction of the reflection coefficient $R_{0}$ of flexural waves from the sharp edge of the wedge covered by an absorbing layer due to the acoustic black hole effect, in agreement with the theory briefly discussed in the previous section. Note that the wedge damping system under consideration is most efficient at medium and high frequencies (above $500 \mathrm{~Hz}$ in this example), simply because the film-induced energy loss factor is more influential at higher frequencies.

Figure 5 shows the effect of increasing the number of the adhesive thin absorbing strips of the same thickness $\delta=0.2 \mathrm{~mm}$ (which is equivalent to the increasing the thickness of the combined absorbing layer) on the measured point mobility of a quadratic wedge. As one can see, the attaching of two or three adhesive strips over each other results in a noticeable reduction of the resonant peaks at medium frequencies (from $500 \mathrm{~Hz}$ to $3000 \mathrm{~Hz}$ ), whereas at higher frequencies the suppression of resonant peaks is rather insignificant. Again, this agrees with the above-mentioned theory of flexural wave reflection from covered wedges of powerlaw profile, which predicts that further increase of an adhesive film thickness relative to the flexural wavelength does not cause significant improvement in the wedge damping properties [4]. The reason for this is that, in spite of the associated increase of the effective wedge-film loss factor, the increase of the film thickness destroys the acoustic black hole effect, thus resulting in a more or less stable damping behaviour of the whole system. This point of view is confirmed also by the measurements of the point mobility of a wedge covered by a thin 
absorbing film of the thickness $\delta=0.2 \mathrm{~mm}$ and by a thick (3-mm) commercial absorbing layer (see Fig. 6). As one can see, the replacement of a thin film by a much thicker layer does not cause much of improvement. This implies that in a wedge damping system employing the acoustic black hole effect it is sufficient to use relatively thin absorbing films, which is very attractive for many practical applications.

One of such potential practical applications can be damping of unwanted resonant vibrations in turbine blades. It is quite obvious that the classical attachment of relatively thick absorbing layers on the whole surface (see e.g. [12]) is incompatible with the main function of a blade. If, however, to make one of the blade edges in the shape materialising the acoustic black hole effect, then even a thin absorbing layer deposited at the very edge of the blade would be able to provide an efficient vibration damping in the whole system. In particular, such a thin absorbing layer could be made of one of the specially developed vibration absorptive and temperature-resistive alloys. One could expect that utilising such alloys would make it possible to manufacture integrated turbine blades with much improved vibration damping performance.

\section{Conclusions}

The reported results of the experimental measurements of point mobility of a free quadratic wedge and of the same wedge covered by adhesive strips of absorbing layers demonstrate a significant suppression of resonant vibration peaks. This can be attributed to the significant reduction of the reflection coefficient of flexural waves from the sharp edge of the wedge, in agreement with the above-mentioned theory utilising the acoustic black hole effect. 
In a wedge damping system employing the acoustic black hole effect it is sufficient to use relatively thin absorbing films, which is a very attractive feature for many practical applications. 


\section{References}

[1] V.V. Krylov, Laminated plates of variable thickness as effective absorbers for flexural vibrations, Proceedings of 17th International Congress on Acoustics, Rome, Italy, 2-7 September 2001, Ed. A. Alippi, Vol. 1 (2001) 270-271.

[2] V.V. Krylov, Acoustic ‘black holes' for flexural waves and their potential applications, Proceedings of the Institute of Acoustics Spring Conference, Salford, UK, 25-27 March 2002 (on CD).

[3] V.V. Krylov, F.J.B.S. Tilman, Acoustic 'black holes' for flexural waves as effective vibration dampers, Journal of Sound and Vibration, 274 (2004) 605-619.

[4] V.V. Krylov, New type of vibration dampers utilising the effect of acoustic 'black holes’, Acta Acustica united with Acustica, 90(5) (2004) 830-837.

[5] C. Vemula, A.N. Norris, Attenuation of waves in plates and bars using a graded impedance interface at edges, Journal of Sound and Vibration, 196 (1996) 107-127.

[6] V.V. Krylov, Conditions for validity of the geometrical-acoustic approximation in application to waves in an acute-angle solid wedge, Soviet Physics - Acoustics, 35 (1989) 176-180. 
[7] V.V. Krylov, Geometrical-acoustics approach to the description of localized vibrational modes of an elastic solid wedge, Soviet Physics - Technical Physics, 35 (1990) 137140.

[8] V.V. Krylov, On the velocities of localised vibration modes in immersed solid wedges, Journal of the Acoustical Society of America, 103 (1998) 767-770.

[9] V.V. Krylov, Localised acoustic modes of a quadratically-shaped solid wedge, Moscow University Physics Bulletin, 45 (1990) 65-69.

[10] V.V. Krylov, A.L. Shuvalov, Propagation of localised flexural vibrations along plate edges described by a power law, Proceedings of the Institute of Acoustics, 22(2) (2000) 263-270.

[11] M.A. Mironov, Propagation of a flexural wave in a plate whose thickness decreases smoothly to zero in a finite interval, Soviet Physics - Acoustics, 34 (1988) 318-319.

[12] D. Ross, E.E. Ungar, E.M. Kerwin, Jr., Damping of plate flexural vibrations by means of viscoelastic laminae, In: J.E. Ruzicka (ed) Structural Damping, Pergamon Press, Oxford 1960, p. 49-87. 


\section{Figure captions}

Fig. 1. Damping of resonant vibrations can be achieved by reducing wave reflections from edges of structures, in addition to a more traditional way of increasing local wave attenuation; in particular, one can apply a non-symmetric quadratic wedge-like damper covered by an absorbing film on one side (a) for suppression of flexural wave reflections from one of the edges of an elastic plate $(b)$ [1-4].

Fig. 2. Experimental set-up.

Fig. 3. Measured point mobility of a free plate; the position of the shaker was at $100 \mathrm{~mm}$ from one of the shorter edges and at $100 \mathrm{~mm}$ from both longer edges; the attachment of adhesive absorbing strips at any edges of the plate did not cause noticeable changes in the frequency response.

Fig. 4. Measured point mobility of a quadratic steel wedge: free wedge (solid curve) and wedge covered by a thin adhesive strip of the thickness $\delta=0.2 \mathrm{~mm}$ (dashed curve); the position of the shaker was at $100 \mathrm{~mm}$ from the thick end of the wedge and at $100 \mathrm{~mm}$ from both side edges; note a significant suppression of resonant peaks in a wedge covered by an adhesive strip.

Fig. 5. Measured point mobility of a quadratic steel wedge covered by several thin adhesive strips of thickness $\delta=0.2 \mathrm{~mm}$ each: one strip (solid curve), two strips (dashed 
curve) and three strips (dash-dotted curve); the position of the shaker was at 100 $\mathrm{mm}$ from the thick end of the wedge and at $100 \mathrm{~mm}$ from both side edges.

Fig. 6. Measured point mobility of a quadratic steel wedge covered by a thin strip of thickness $\delta=0.2 \mathrm{~mm}$ (dashed curve) and by a thick (3-mm) adhesive layer (solid curve); the position of the shaker was at $100 \mathrm{~mm}$ from the thick end of the wedge and at $100 \mathrm{~mm}$ from both side edges. 


\section{Figures}

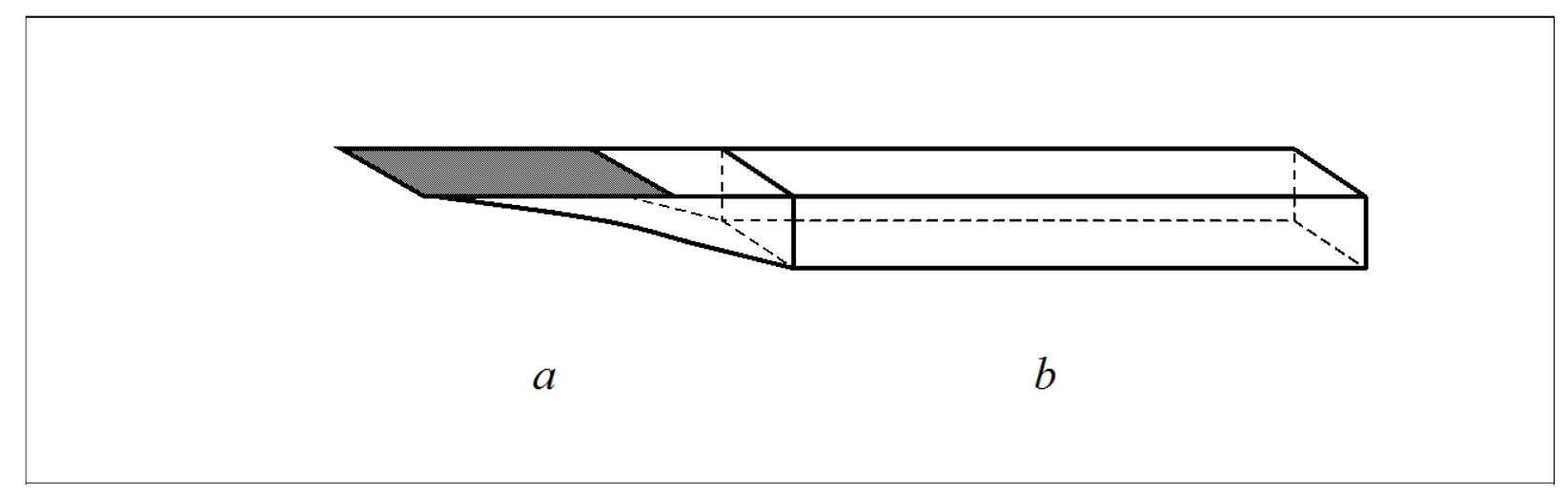

Fig. 1. Damping of resonant vibrations can be achieved by reducing wave reflections from edges of structures, in addition to a more traditional way of increasing local wave attenuation; in particular, one can apply a non-symmetric quadratic wedge-like damper covered by an absorbing film on one side (a) for suppression of flexural wave reflections from one of the edges of an elastic plate (b) [1-4]. 


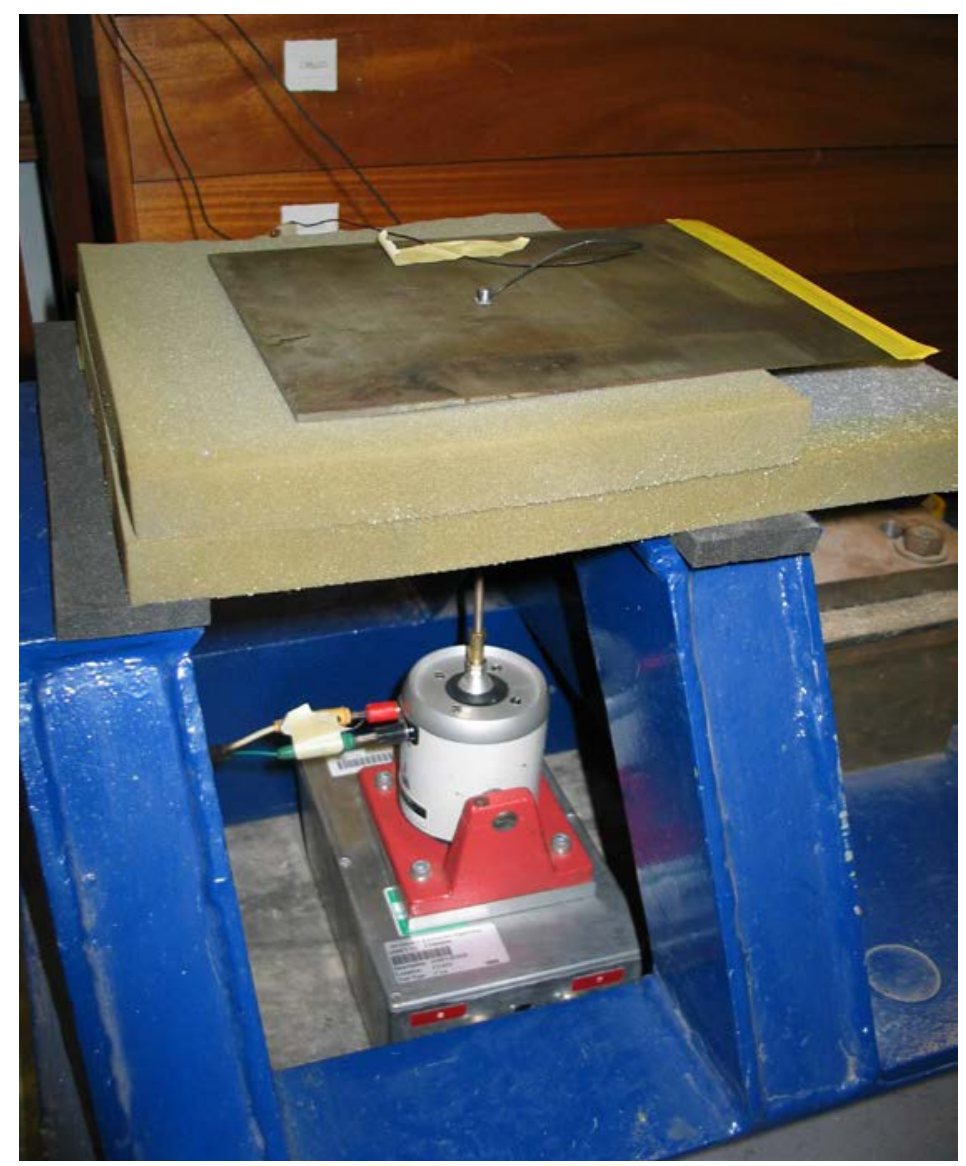

Fig. 2. Experimental set-up. 


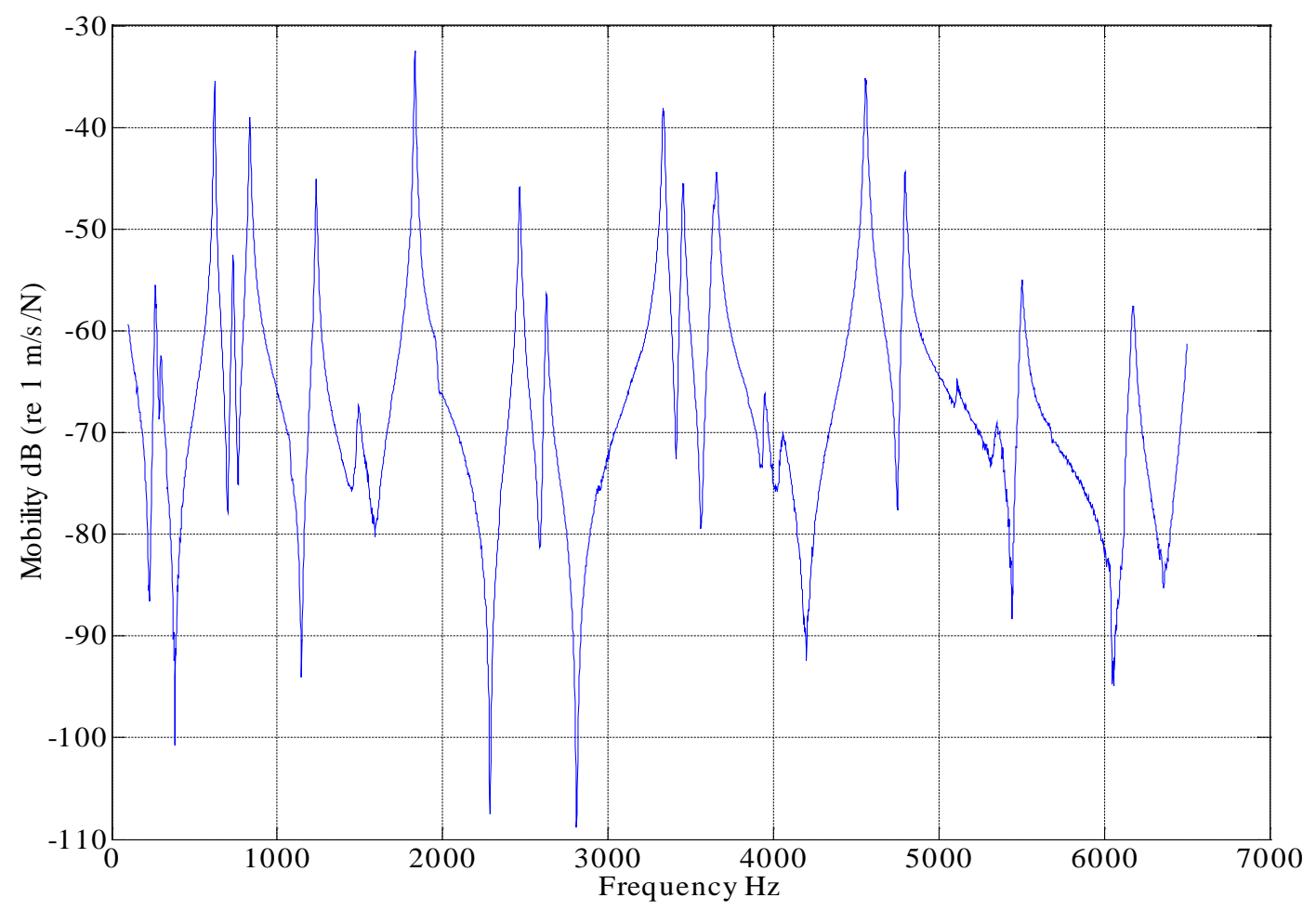

Fig. 3. Measured point mobility of a free plate; the position of the shaker was at $100 \mathrm{~mm}$ from one of the shorter edges and at $100 \mathrm{~mm}$ from both longer edges; the attachment of adhesive absorbing strips at any edges of the plate did not cause noticeable changes in the frequency response. 


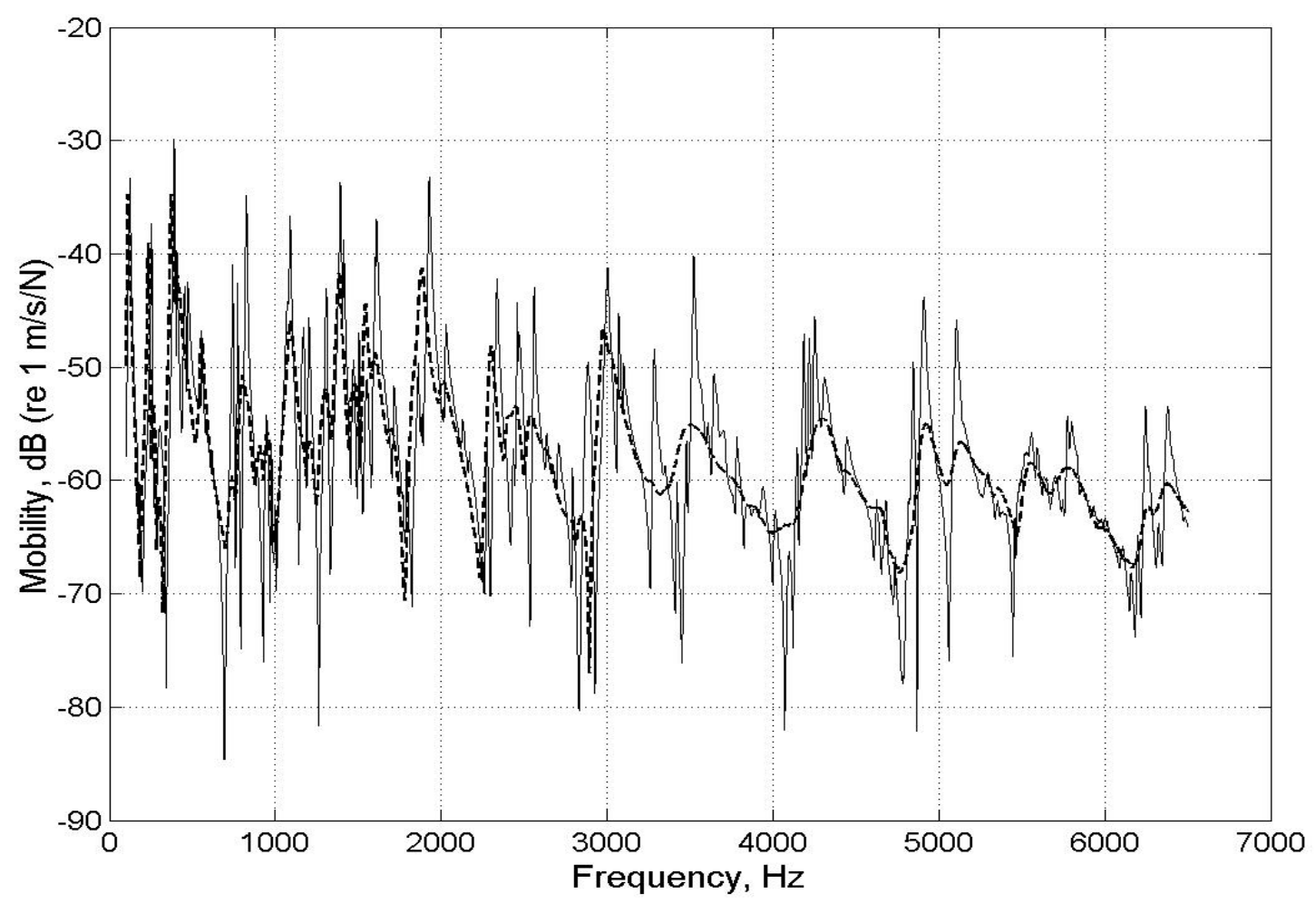

Fig. 4. Measured point mobility of a quadratic steel wedge: free wedge (solid curve) and wedge covered by a thin adhesive strip of the thickness $\delta=0.2 \mathrm{~mm}$ (dashed curve); the position of the shaker was at $100 \mathrm{~mm}$ from the thick end of the wedge and at $100 \mathrm{~mm}$ from both side edges; note a significant suppression of resonant peaks in a wedge covered by an adhesive strip. 


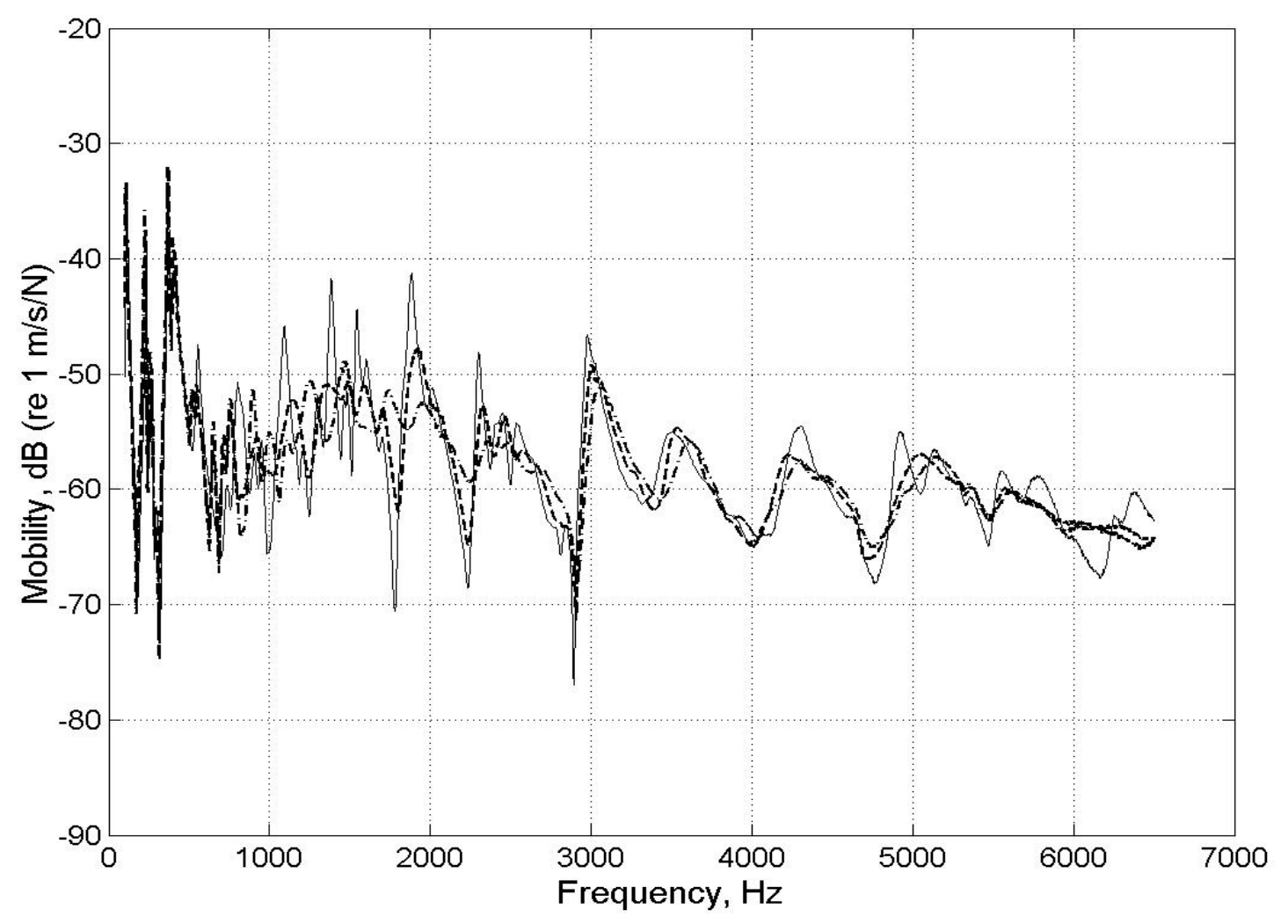

Fig. 5. Measured point mobility of a quadratic steel wedge covered by several thin adhesive strips of thickness $\delta=0.2 \mathrm{~mm}$ each: one strip (solid curve), two strips (dashed curve) and three strips (dash-dotted curve); the position of the shaker was at $100 \mathrm{~mm}$ from the thick end of the wedge and at $100 \mathrm{~mm}$ from both side edges. 


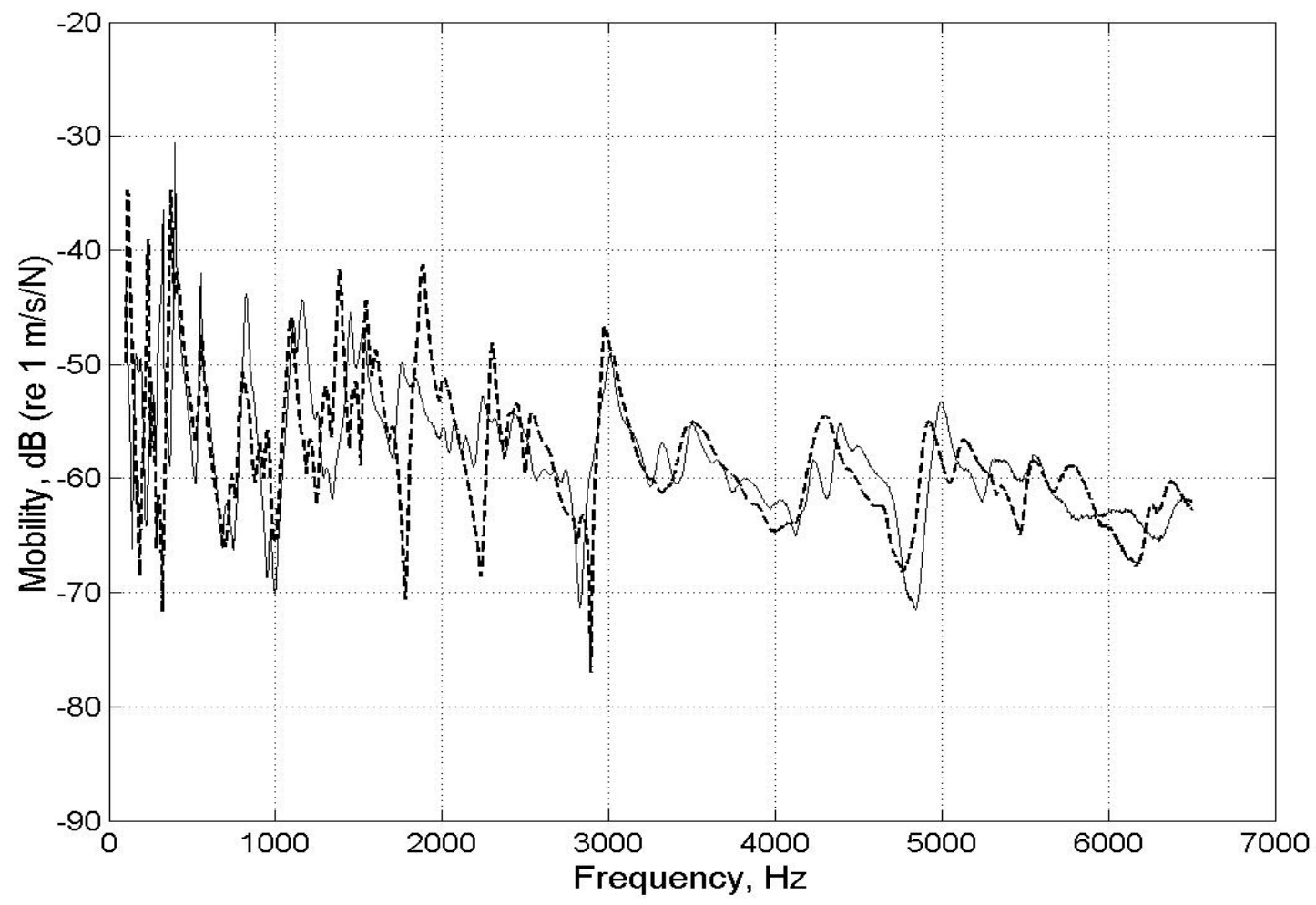

Fig. 6. Measured point mobility of a quadratic steel wedge covered by a thin strip of thickness $\delta=0.2 \mathrm{~mm}$ (dashed curve) and by a thick (3$\mathrm{mm}$ ) adhesive layer (solid curve); the position of the shaker was at $100 \mathrm{~mm}$ from the thick end of the wedge and at $100 \mathrm{~mm}$ from both side edges. 